\title{
Nonlinear SpatioTemporal Channel Gain Map Tracking in Mobile Cooperative Networks
}

\author{
Dionysios S. Kalogerias ${ }^{\dagger}$ and Athina P. Petropulu \\ Department of Electrical \& Computer Engineering, \\ Rutgers, The State University of New Jersey, Piscataway, NJ, USA \\ e-mail: \{d.kalogerias, athinap\} @ rutgers.edu
}

\begin{abstract}
We propose a nonlinear filtering framework for channel state tracking and spatiotemporal channel gain prediction in mobile wireless sensor networks, in a Bayesian setting. Under common assumptions, the wireless channel constitutes an observable (by the sensors/network nodes), spatiotemporal, conditionally Gaussian stochastic process, which is statistically dependent on a set of hidden channel parameters, called the channel state. The channel state evolves in time according to a known, non Gaussian Markov stochastic kernel. Advocating the use of grid based approximate filters as an effective and robust means for recursive tracking of the channel state, we propose a sequential spatiotemporal predictor for tracking the respective channel gain map, for each sensor in the network. We also show that both estimators (state and gain map trackers) converge towards the true respective Minimum Mean Squared Error (MMSE) optimal estimators, in a common, relatively strong sense. Numerical simulations corroborate the practical effectiveness of the proposed approach.
\end{abstract}

Index Terms-Mobile Wireless Networks, Channel Gain Maps, Spatiotemporal Channel Prediction, Nonlinear Filtering, Sequential Estimation.

\section{INTRODUCTION}

Recent advances in wireless networks and distributed communication and processing systems has led to the formulation of new, challenging problems, related not only to the flow of information over networks, but also to the estimation and control of the underlying physical layer. In a number of important applications (see, e.g., [1], [2]), accurate estimation of Channel State Information (CSI), or Statistical CSI (SCSI), for all of the nodes/sensors in a wireless network is essential. Traditionally, in such applications, CSI and SCSI estimation is done via pilot based schemes, blind channel estimation techniques, or even through averaging from rough channel observations. Except for the fact that naive extension of the conventional techniques to larger scale wireless networks requires collaboration between the network nodes, which can be both bandwidth and power intensive, these techniques are only sufficient for relatively lower rate and/or quasistatic environments, where the statistics of the communication medium do not change significantly over time. However, the behavior of most indoor and outdoor communication environments of practical interest is intrinsically time varying (see, e.g., [3]).

In addition to the temporal variation of the wireless medium, recently, its spatial variation has attracted considerable interest as well. In fact, learning how the communication channel

${ }^{\dagger}$ Corresponding Author.

This work is supported by the National Science Foundation (NSF) under grant CNS - 1239188. evolves through space is tightly connected to the ability of a network to assess the quality of the channel at previously unexplored locations in space. Such knowledge would be highly beneficial in a number of new and important applications, such as mobile beamforming [4] and mobility enhanced physical layer security [5], [6], in which both transmission weights and node locations become decision variables.

To the best of our knowledge, the first systematic approach to joint spatiotemporal channel (shadowing) tracking and prediction was recently presented in [7], [8], where the use of Channel Gain (CG) maps was advocated as an advantageous alternative to Power Spectral Density (PSD) maps for cooperative spectrum sensing in the context of cognitive radios. However, although analytically appealing, the state space model considered in [7], [8] for describing the spatiotemporal evolution of the wireless channel is rather restrictive; both the dependence of the shadowing field on its previous value in time and its spatial interactions are characterized by purely linear functional relationships, focusing mainly on modeling the spatiotemporal variations of the trend of the field.

In this work, in order to facilitate conceptualization, we consider a simple network configuration, comprised by a "reference" point/antenna, capable of broadcasting global information, as well as a set of possibly mobile network nodes/sensors, capable either of local message exchange, or communicating with a fusion center. The statistical model describing the joint spatiotemporal behavior of the channel measurements gathered at the sensors is inspired by [9]. However, different from [9], the descriptive channel parameters (e.g., the path loss exponent, the shadowing power, etc.), referred to here as the channel state, are assumed to be temporally varying. Specifically, we assume that the whole channel state constitutes a Markov process, with known, but potentially nonlinear and/or non Gaussian transition model. Essentially, the spatiotemporal evolution of the channel is conveniently modeled as a partially observable dynamical system (with Markovian dynamics), more commonly referred to as a Hidden Markov Model (HMM) [10].

Our main contributions are as follows: 1) Recognizing the obvious intractability of state estimation in partially observable nonlinear systems, we propose the use of grid based approximate nonlinear recursive filters for sequential channel state tracking, based on the so called marginal approximations of the channel state [11]. Then, exploiting filtered estimates of the channel state, a recursive spatiotemporal predictor of the channel gains (magnitudes) 
is developed, providing real time sequential estimates for the respective CG map, for each sensor in the network. 2) We provide a set of simple, relaxed conditions, under which the proposed channel state tracker is asymptotically optimal, in the sense that it converges to the respective true Minimum Mean Squared Error (MMSE) channel state estimator, in a relatively strong sense. The convergence of the proposed spatiotemporal predictor is established in exactly the same sense, providing a unified convergence criterion for both sequential estimators.

\section{System Model \& PROBlem Formulation}

For simplicity of exposition, we consider a wireless network described as follows. The environment is assumed to be a closed planar region $\mathcal{S} \subset \mathbb{R}^{2}$, where there exists a fixed, stationary antenna at a reference position, capable of at least information broadcasting. There also exist a set of $N$ single antenna sensors, possibly mobile, monitoring the channel relative to the reference antenna. The sensors can cooperate and can either communicate with a fusion center (in a centralized setting), or exchange basic messages amongst each other (in a decentralized/infrastructureless scenario) using a low rate dedicated channel. Concerning channel modeling, we adopt a flat fading model between each node and the reference antenna. It is additionally assumed that channel reciprocity holds and that all network nodes can perfectly observe their individual channel realizations (e.g. magnitudes and potentially phases) relative to the reference antenna [9].

Let us describe our problem in more explicit mathematical terms. All stochastic processes are defined on a common probability triplet $(\Omega, \mathscr{F}, \mathcal{P})$. Let $X_{t} \equiv X_{t}(\omega) \subset \mathbb{R}^{M \times 1}$, $t \in \mathbb{N}, \omega \in \Omega$ denote the hidden channel state. Under the flat fading assumption, the relative to the reference antenna complex channel process at each network node $i \in \mathbb{N}_{N}^{+}$, located at $\mathbf{p}_{i} \equiv \mathbf{p}_{i}(t) \in \mathcal{S}$ (that is, the nodes might be moving), can be decomposed as [12]

$$
\begin{aligned}
\mathbb{C} \ni Y_{i}\left(\mathbf{p}_{i}(t), X_{t}\right) \equiv Y_{\mathbf{p}_{i}}\left(X_{t}\right) \equiv \\
\underbrace{Y_{\mathbf{p}_{i}}^{P L}\left(X_{t}\right)}_{\text {path loss }} \underbrace{Y_{i}^{S H}\left(X_{t}\right)}_{\text {shadowing }} \underbrace{Y_{\mathbf{p}_{i}}^{M F}(t) \exp \left(\mathfrak{J} \frac{2 \pi d_{i}(t)}{\lambda}\right)}_{\text {fading }},
\end{aligned}
$$

where $\mathfrak{J} \triangleq \sqrt{-1}, \lambda \in \mathbb{R}_{++}$denotes the wavelength employed for the communication and where: 1) $Y_{\mathbf{p}_{i}}^{P L}\left(X_{t}\right) \in \mathbb{R}$ denotes path loss, defined as $Y_{\mathbf{p}_{i}}^{P L}\left(X_{t}\right) \triangleq\left\|\mathbf{p}_{i}(t)-\mathbf{p}_{r e f}\right\|_{2}^{-\mu\left(X_{t}\right) / 2} \triangleq$ $\left(d_{i}(t)\right)^{-\mu\left(X_{t}\right) / 2}$, where $\mu\left(X_{t}\right) \in \mathbb{R}_{++}$is the state dependent path loss exponent, which is the same for all network nodes and $\mathbf{p}_{r e f} \in \mathcal{S}$ denotes the position of the reference antenna in $\mathcal{S}$. 2) $Y_{i}^{S H}\left(X_{t}\right) \in \mathbb{R}$ denotes the shadowing part of the channel model and its square, conditionally on $X_{t}$, constitutes a base-10 log-normal random variable with zero location and scale depending on $X_{t}$. 3) $Y_{\mathbf{p}_{i}}^{M F}(t) \in \mathbb{C}$ represents multipath fading, which is assumed to be a spatiotemporally white [9], strictly stationary process with fully known statistical description, not associated with $X_{t}$, therefore being an unpredictable complex "observation noise". Making the substitution $Y_{i}\left(\mathbf{p}_{i}(t), X_{t}\right) \leftarrow \exp \left(-\mathfrak{J} 2 \pi d_{i}(t) / \lambda\right) Y_{i}\left(\mathbf{p}_{i}(t), X_{t}\right)$, we can define the observations of the $i$-th node in $d B$ scale as [13]

$$
y_{t}^{i} \triangleq \alpha_{t}^{i} \mu\left(X_{t}\right)+\sigma_{t}^{i}\left(X_{t}\right)+\xi_{t}^{i},
$$

where, $\forall t \in \mathbb{N}$ and $\forall i \in \mathbb{N}_{N}^{+}, \alpha_{t}^{i} \triangleq 10 \log _{10}\left(d_{i}(t)\right), \sigma_{t}^{i}\left(X_{t}\right) \triangleq$ $10 \log _{10}\left(Y_{i}^{S H}\left(X_{t}\right)\right)^{2}$ and $\xi_{t}^{i} \triangleq \overline{10 \log _{10}\left|Y_{\mathbf{p}_{i}}^{M F}(t)\right|^{2}}$ and where the quantity $-10 \mathbb{E}\left\{\log _{10}\left|Y_{\mathbf{p}_{i}}^{M F}(t)\right|^{2}\right\}$ has been subtracted from the left hand side of (2) $(\overline{(\cdot)}$ denotes the zero mean version of a random variable). We should emphasize here that by "measurement" or "observation" we refer to the predictable component of the channel, which is described in terms of the channel magnitude.

The following further assumptions are made [9]: $\xi_{t}^{i} \stackrel{i . i . d .}{\sim}$ $\mathcal{N}\left(0, \sigma_{\xi}^{2}\right), \forall i \in \mathbb{N}_{N}^{+}$and $\forall t \in \mathbb{N}$. Second, conditional on $X_{t}, \sigma_{t}^{i}\left(X_{t}\right) \stackrel{i . d .}{\sim} \mathcal{N}\left(0, \eta^{2}\left(X_{t}\right)\right), \forall i \in \mathbb{N}_{N}^{+}$. Additionally, for each set of positions of the network nodes in $\mathcal{S}$, the members of the set $\left\{\sigma_{t}^{i}\left(X_{t}\right)\right\}_{i \in \mathbb{N}_{N}^{+}}$constitute jointly normal, spatially correlated random variables with conditional on $X_{t}$ autocorrelation kernel [14] $\mathcal{R}_{i j}\left(X_{t}\right) \triangleq$ $\eta^{2}\left(X_{t}\right) \exp \left(-d_{i j}(t) / \beta\left(X_{t}\right)\right)$, for all $(i, j) \in \mathbb{N}_{N}^{+} \times \mathbb{N}_{N}^{+}$, where $d_{i j}(t) \triangleq\left\|\mathbf{p}_{i}(t)-\mathbf{p}_{j}(t)\right\|_{2} \in \mathbb{R}_{+}$and $\beta\left(X_{t}\right) \in \mathbb{R}_{++}$. Therefore, $\mathcal{R}_{i j}\left(X_{t}\right)$ directly defines the $(i \cdot j)$-th entry of the time evolving, conditional (on $X_{t}$ ) covariance matrix of the random vector $\boldsymbol{\sigma}_{t}\left(X_{t}\right) \triangleq\left[\left\{\sigma_{t}^{i}\left(X_{t}\right)\right\}_{i \in \mathbb{N}_{N}^{+}}\right]^{\boldsymbol{T}} \in \mathbb{R}^{N \times 1}$, $\boldsymbol{\Sigma}_{t}\left(X_{t}\right) \triangleq \mathbb{E}\left\{\boldsymbol{\sigma}_{t}\left(X_{t}\right) \boldsymbol{\sigma}_{t}^{\boldsymbol{T}}\left(X_{t}\right) \mid X_{t}\right\} \in \mathcal{D}_{\boldsymbol{\Sigma}} \subset \mathbb{R}^{N \times N}$, with $\mathcal{D}_{\Sigma}$ bounded. In the following, for mathematical simplicity, although there are endless possibilities for defining the state dependent functions $\mu\left(X_{t}\right), \eta^{2}\left(X_{t}\right)$ and $\beta\left(X_{t}\right)$, we will assume that $\mu\left(X_{t}\right) \equiv X_{t}(1), \eta^{2}\left(X_{t}\right) \equiv X_{t}(2)$ and $\beta\left(X_{t}\right) \equiv X_{t}(3)$, also in agreement with our intuition.

In order to define an overall observation process for all nodes in the network, we may stack the $N$ individual channel processes of (2), resulting in the vector observation model

$$
\mathbf{y}_{t} \equiv \boldsymbol{A}_{t} X_{t}+\boldsymbol{\sigma}_{t}\left(X_{t}\right)+\boldsymbol{\xi}_{t}, \quad \forall t \in \mathbb{N},
$$

where $\boldsymbol{\sigma}_{t}\left(X_{t}\right)$ is defined as above, $\boldsymbol{A}_{t} \triangleq\left[\boldsymbol{\alpha}_{t} \mathbf{0}_{N \times(M-1)}\right] \in$ $\mathbb{R}^{N \times M}$ and with $\mathbf{y}_{t} \in \mathbb{R}^{N \times 1}, \boldsymbol{\alpha}_{t} \in \mathbb{R}^{N \times 1}$ and $\boldsymbol{\xi}_{t} \in$ $\mathbb{R}^{N \times 1}$ defined accordingly. The observation process (3) can also be rewritten in the canonical form $\mathbf{y}_{t} \equiv \boldsymbol{\alpha}_{t} \mu\left(X_{t}\right)+$ $\sqrt{\mathbf{C}_{t}\left(X_{t}\right)} \boldsymbol{u}_{t}, \forall t \in \mathbb{N}$, where $\boldsymbol{u}_{t} \equiv \boldsymbol{u}_{t}(\omega)$ constitutes a standard Gaussian white noise process and $\mathbf{C}_{t}\left(X_{t}\right) \triangleq \boldsymbol{\Sigma}_{t}\left(X_{t}\right)+$ $\sigma_{\xi}^{2} \mathbf{I}_{N \times N} \in \mathcal{D}_{\mathbf{C}}$, with $\mathcal{D}_{\mathbf{C}}$ obviously bounded.

Regarding the underlying channel state process $X_{t} \in$ $\mathbb{R}^{M \times 1}$, we will assume that it constitutes a Markov process with known but nonlinear and (for simplicity) stationary dynamics, described by a stochastic kernel $\mathcal{K}: \mathscr{B}\left(\mathbb{R}^{M \times 1}\right) \times$ $\mathbb{R}^{M \times 1} \mapsto[0,1]$. Also, we will make the generic and realistic assumption that the state is confined to a compact strict subset of $\mathbb{R}^{M \times 1}$, that is, $\forall t \in \mathbb{N}, X_{t} \in[a, b]^{M} \triangleq \mathcal{Z} \subset \mathbb{R}^{M \times 1}$, almost surely. In addition to the above and for technical reasons [13], [15], [16] we assume that $\lambda_{\text {inf }} \equiv \inf _{t \in \mathbb{N}} \inf _{\boldsymbol{x} \in \mathcal{Z}} \lambda_{\min }\left(\mathbf{C}_{t}(\boldsymbol{x})\right)>1$, a requirement which can always be satisfied by appropriate 
normalization of the observations.

Hereafter, strict optimality ${ }^{1}$ will imply optimality in the Minimum Mean Squared Error (MMSE) sense. Also, the natural filtration generated by the causal observation process $\mathbf{y}_{t}$ is denoted as $\left\{\mathscr{Y}_{t}\right\}_{t \in \mathbb{N}}$, where $\mathscr{Y}_{t} \triangleq \sigma\left\{\left\{\mathbf{y}_{i}\right\}_{i \in \mathbb{N}_{t}}\right\}, \forall t \in \mathbb{N}$. Here, $\sigma\{A\}$ denotes the $\sigma$-algebra generated by the random element $A$. The problems of interest in this paper are defined as follows.

Problem 1. (Sequential Channel State Tracking (SCST)) Develop a theoretically grounded, fixed complexity ${ }^{2}$ sequential scheme for (approximately) evaluating the strictly optimal filter or $\rho$-step predictor of the channel state $X_{t}$ on the basis of the available channel magnitude observations up to time $t$, given by

$$
\widehat{X}_{t+\rho} \triangleq \mathbb{E}\left\{X_{t+\rho} \mid \mathscr{Y}_{t}\right\}, \quad \forall t \in \mathbb{N},
$$

where $\rho \geq 0$ constitutes the prediction horizon.

Problem 2. (Sequential Spatiotemporal Channel Prediction (SSCP)) Develop a theoretically grounded, fixed complexity sequential scheme for (approximately) evaluating the strictly optimal spatiotemporal predictor of the channel magnitude at position $\mathbf{q} \in \mathbb{R}^{2}$ and time $t+\rho(\rho \geq 0$ is the prediction horizon), given the available channel magnitude observations up to time $t$, expressed as

$$
\widehat{y}_{t+\rho}(\mathbf{q}) \triangleq \mathbb{E}\left\{y_{t+\rho}(\mathbf{q}) \mid \mathscr{Y}_{t}\right\}, \quad \forall t \in \mathbb{N} .
$$

\section{APPROXIMATE Filtering OF MARKOV PROCESSES}

In the following, we present a number of preliminary results in asymptotically optimal, approximate recursive filtering of Markov processes, recently presented in [15].

\section{A. Uniform State Quantizations}

From Section II, we have assumed that $X_{t} \in \mathcal{Z} \equiv$ $[a, b]^{M}, \forall t \in \mathbb{N}$, a.s., where, geometrically, $\mathcal{Z}$ constitutes an $M$-hypercube, representing the compact set of support of the state $X_{t}$. Let us discretize $\mathcal{Z}$ into $L_{S} \triangleq L^{M}$ hypercubic $M$ dimensional cells of identical volume (each dimension is partitioned in to $L$ intervals). The center of mass of the $l$-th cell is denoted as $\boldsymbol{x}_{L_{S}}^{l}, l \in \mathbb{N}_{L_{S}}^{+}$. Then, letting $\mathcal{X}_{L_{S}} \triangleq\left\{\boldsymbol{x}_{L_{S}}^{l}\right\}_{l \in \mathbb{N}_{L_{S}}^{+}}$, the quantizer $\mathcal{Q}_{L_{S}}:(\mathcal{Z}, \mathscr{B}(\mathcal{Z})) \mapsto\left(\mathcal{X}_{L_{S}}, 2^{\mathcal{X}_{L_{S}}}\right)$ is defined as the bijective and measurable function which uniquely maps the $l$-th cell to the respective reconstruction point $\boldsymbol{x}_{L_{S}}^{l}$, $\forall l \in \mathbb{N}_{L_{S}}^{+}$, according to some predefined ordering. That is, $\mathcal{Q}_{L_{S}}(\boldsymbol{x}) \triangleq \boldsymbol{x}_{L_{S}}^{l}$ if and only if $\boldsymbol{x}$ belongs to the respective cell (for a detailed and more formalistic definition, see [15]). Having defined the quantizer $\mathcal{Q}_{L_{S}}(\cdot)$, the Marginal Quantization of the state is defined as [11]

$$
X_{t}^{L_{S}}(\omega) \triangleq \mathcal{Q}_{L_{S}}\left(X_{t}(\omega)\right) \in \mathcal{X}_{L_{S}}, \forall t \in \mathbb{N}, \mathcal{P}-\text { a.s. }
$$

\footnotetext{
${ }^{1}$ In this paper, an optimal estimator is referred to as "strictly optimal", in order to emphasize its difference compared to an approximately/asymptotically optimal one, in the same, of course, sense.

${ }^{2}$ The term "fixed complexity" means that the computational cost does not grow with time.
}

where $L_{S} \in \mathbb{N}$ is identified as the approximation parameter. That is, $X_{t}$ is approximated by its nearest neighbor on the cell grid. Additionally, for later reference, define the column stochastic matrix $\boldsymbol{P} \in[0,1]^{L_{S} \times L_{S}}$ as $\boldsymbol{P}(i, j) \triangleq$ $\mathcal{P}\left(X_{t}^{L_{S}} \equiv \boldsymbol{x}_{L_{S}}^{i} \mid X_{t-1}^{L_{S}} \equiv \boldsymbol{x}_{L_{S}}^{j}\right), \forall(i, j) \in \mathbb{N}_{L_{S}}^{+} \times \mathbb{N}_{L_{S}}^{+}$. Due to its structure, $\boldsymbol{P}$ can at least be constructed simulating $X_{t}^{L_{S}}$, by the Law of Large Numbers. Note, however, that it is also possible to obtain $\boldsymbol{P}$ using only available realizations of the state, without actually knowing the stochastic kernel of $X_{t}$. For example, this could be made possible in sufficiently controlled physical experiments, where the channel state $X_{t}$ would be a fully observable stochastic process.

\section{B. Asymptotically Optimal Recursive Estimators}

First, let us introduce the useful concept of conditional regularity of stochastic kernels/processes.

Definition 1. (Conditional Regularity of Stochastic Kernels [15]) Consider the stochastic (or Markov) kernel $\mathcal{K}$ : $\mathscr{B}\left(\mathbb{R}^{M \times 1}\right) \times \mathbb{R}^{M \times 1} \mapsto[0,1]$, associated with the process $Y_{t}(\omega) \in \mathbb{R}^{M \times 1}$, for all $t \in \mathbb{N}$. We say that $\mathcal{K}(\cdot \mid \cdot)$ is Conditionally Regular of Type I (CRT I), if, for almost all $\boldsymbol{x} \in \mathcal{Z}$, there exists a bounded sequence $\delta_{n}(\boldsymbol{x}) \in \mathbb{R}_{+}, n \in \mathbb{N}^{+}$ such that, for $\mathcal{A} \in\left\{\mathcal{Z}_{L_{S}}^{j}\right\}_{j \in \mathbb{N}_{L_{S}}^{+}}$,

$$
\sup _{\mathcal{A}}\left|\mathcal{K}(\mathcal{A} \mid \boldsymbol{x})-\mathcal{K}\left(\mathcal{A} \mid \mathcal{Q}_{L_{S}}(\boldsymbol{x})\right)\right| \leq \frac{\delta_{L_{S}}(\boldsymbol{x})}{L_{S}} \underset{L_{S} \rightarrow \infty}{\stackrel{\text { a.e. }}{\rightarrow}} 0 .
$$

If, additionally, for almost all $\boldsymbol{x} \in \mathbb{R}^{M \times 1}$, the Borel probability measure $\mathcal{K}(\cdot \mid \boldsymbol{x})$ admits a stochastic kernel density $\kappa: \mathbb{R}^{M \times 1} \times \mathbb{R}^{M \times 1} \mapsto \mathbb{R}_{+}$, suggestively denoted as $\kappa(\boldsymbol{y} \mid \boldsymbol{x})$ and if the condition

$$
\sup _{\boldsymbol{y} \in \mathbb{R}^{M \times 1}}\left|\kappa(\boldsymbol{y} \mid \boldsymbol{x})-\kappa\left(\boldsymbol{y} \mid \mathcal{Q}_{L_{S}}(\boldsymbol{x})\right)\right| \leq \delta_{L_{S}}(\boldsymbol{x}) \underset{L_{S} \rightarrow \infty}{\stackrel{a . e_{j}}{\longrightarrow}} 0
$$

is satisfied, then we say that $\mathcal{K}(\cdot \mid \cdot)$ is Conditionally Regular of Type II (CRT II). In any of the two cases, we will also say that $Y_{t}$ is conditionally regular, interchangeably.

Having defined conditional regularity, the following result is true, showing that it is possible to approximate the strictly optimal nonlinear filter/predictor $\widehat{X}_{t+\rho}$ by a simple recursive filtering scheme, being formally similar to the MMSE optimal filter of a Markov chain with finite state space. Further, it turns out that the resulting approximate filter is asymptotically consistent in a relatively strong sense.

In the following, $\mathcal{Q}_{L_{S}}^{e}:\left(\mathcal{X}_{L_{S}}, 2^{\mathcal{X}_{L_{S}}}\right) \mapsto\left(\mathcal{B}_{L_{S}}, 2^{\mathcal{B}_{L_{S}}}\right)$ constitutes a unique bijective mapping between the sets $\mathcal{X}_{L_{S}}$ and $\mathcal{B}_{L_{S}} \triangleq\left\{\mathbf{e}_{l}^{L_{S}}\right\}_{l \in \mathbb{N}_{L_{S}}^{+}}$, where the latter contains as elements the complete standard basis in $\mathbb{R}^{L_{S} \times 1}$.

Theorem 1. (Approximate Filtering of Markov Processes [13], [15]) Define the reconstruction and likelihood matrices as $\mathbf{X} \triangleq\left[\boldsymbol{x}_{L_{S}}^{1} \boldsymbol{x}_{L_{S}}^{2} \ldots \boldsymbol{x}_{L_{S}}^{L_{S}}\right] \in \mathbb{R}^{M \times L_{S}}$ and $\boldsymbol{\Lambda}_{t} \triangleq$ $\operatorname{diag}\left(\left\{\lambda_{t}\left(\boldsymbol{x}_{L_{S}}^{j}\right)\right\}_{j \in \mathbb{N}_{L_{S}}^{+}}\right) \in \mathbb{R}^{L_{S} \times L_{S}}$, respectively, where, 


$$
\lambda_{t}\left(\boldsymbol{x}_{L_{S}}^{j}\right) \triangleq \frac{\exp \left(-\frac{1}{2}\left(\mathbf{y}_{t}-\boldsymbol{A}_{t} \boldsymbol{x}_{L_{S}}^{j}\right)^{\boldsymbol{T}}\left(\boldsymbol{\Sigma}_{t}\left(\boldsymbol{x}_{L_{S}}^{j}\right)+\sigma_{\xi}^{2} \mathbf{I}_{N \times N}\right)^{-1}\left(\mathbf{y}_{t}-\boldsymbol{A}_{t} \boldsymbol{x}_{L_{S}}^{j}\right)\right)}{\sqrt{\operatorname{det}\left(\boldsymbol{\Sigma}_{t}\left(\boldsymbol{x}_{L_{S}}^{j}\right)+\sigma_{\xi}^{2} \mathbf{I}_{N \times N}\right)}} \in \mathbb{R}_{++}, \quad \forall j \in \mathbb{N}_{L_{S}}^{+}
$$

for all $t \in \mathbb{N}, \lambda_{t}\left(\boldsymbol{x}_{L_{S}}^{j}\right)$ is given by (6) (top of page). Then, the strictly optimal filter and $\rho$-step predictor of the state process $X_{t}$ can be approximated as

$$
\mathcal{E}^{L_{S}}\left(X_{t+\rho} \mid \mathscr{Y}_{t}\right) \triangleq \mathbf{X} \boldsymbol{P}^{\rho} E_{t} /\left\|E_{t}\right\|_{1}, \quad \forall t \in \mathbb{N}
$$

and for all finite prediction horizons $\rho \geq 0$, where the process $E_{t} \in \mathbb{R}^{L_{S} \times 1}$ on the RHS of (8) satisfies the simple linear recursion

$$
E_{t} \equiv \boldsymbol{\Lambda}_{t} \boldsymbol{P} E_{t-1}, \quad \forall t \in \mathbb{N},
$$

where $E_{-1} \equiv \mathbb{E}\left\{\mathcal{Q}_{L_{S}}^{e}\left(X_{-1}^{L_{S}}\right)\right\}$. Further, suppose that $X_{t}$ is conditionally regular. Then, for any finite prediction horizon $\rho \geq 0$, there exists a measurable subset $\widehat{\Omega}_{T} \subseteq \Omega$ with $\mathcal{P}$ measure at least $1-(T+1)^{1-C N} \exp (-C N)$, such that

$$
\sup _{t \in \mathbb{N}_{T}} \sup _{\omega \in \widehat{\Omega}_{T}}\left\|\mathcal{E}^{L_{S}}\left(X_{t+\rho} \mid \mathscr{Y}_{t}\right)-\widehat{X}_{t+\rho}\right\|_{1}(\omega) \underset{L_{S} \rightarrow \infty}{\longrightarrow} 0,
$$

for any free, finite constant $C \geq 1$. In other words, the convergence of the respective approximate filters is compact in $t \in \mathbb{N}$ and, with probability at least $1-(T+1)^{1-C N} \exp (-C N)$, uniform in $\omega$.

\section{SCST \& SSCP IN Mobile WiRELESS NETWORKS}

At this point, it is apparent that Theorem 1 in fact directly provides us with an effective approximate and recursive estimator for the channel state $X_{t}$. Therefore, Theorem 1 immediately solves the SCST problem, since the resulting filtering/prediction scheme is sequential and, as new channel measurements become available, its computational complexity is fixed, due to time invariance of the type of numerical operations required for each filter update.

Next, focusing on the SSCP problem, let $\alpha^{\mathrm{q}} \triangleq$ $-10 \log _{10}\left(\left\|\mathbf{q}-\mathbf{p}_{\text {ref }}\right\|_{2}\right), \sigma^{\mathbf{q}}\left(X_{t}\right) \mid X_{t} \stackrel{\text { def }}{\sim} \mathcal{N}\left(0, \eta^{2}\left(X_{t}\right)\right)$ and $\xi^{\mathbf{q}} \stackrel{\text { def }}{\sim} \mathcal{N}\left(0, \sigma_{\xi}^{2}\right)$, where each element of $\gamma_{t}^{\mathbf{q}}\left(X_{t}\right) \triangleq$ $\left[\left\{\mathbb{E}\left\{\sigma^{\mathbf{q}}\left(X_{t}\right) \sigma_{t}^{i}\left(X_{t}\right)\right\}\right\}_{i \in \mathbb{N}_{N}^{+}}\right]^{\boldsymbol{T}} \in \mathbb{R}^{N \times 1}$ is given by $\boldsymbol{\gamma}_{t}^{\mathbf{q}}\left(X_{t}\right)(j) \equiv \eta^{2}\left(X_{t}\right) \exp \left(-\left\|\mathbf{q}-\mathbf{p}_{j}(t)\right\|_{2} / \beta\left(X_{t}\right)\right), \forall j \in$ $\mathbb{N}_{N}^{+}$. Then, it must be true that $y_{t}(\mathbf{q}) \equiv A^{\mathbf{q}} X_{t}+\sigma^{\mathbf{q}}\left(X_{t}\right)+\xi^{\mathbf{q}}$, where $A^{\mathbf{q}} \triangleq\left[\alpha^{\mathbf{q}} \mathbf{0}_{1 \times(M-1)}\right] \in \mathbb{R}^{1 \times M}$, since $y_{t}(\mathbf{q})$ can be equivalently considered as an additional observation, measured by an imaginary sensor at position $\mathbf{q}$. Then, it can be easily shown that [13] $\widehat{y}_{t}(\mathbf{q}) \equiv \mathbb{E}\left\{\phi_{t}\left(X_{t}, \mathbf{y}_{t}\right) \mid \mathscr{Y}_{t}\right\}$, where

$$
\begin{aligned}
& \phi_{t}\left(X_{t}, \mathbf{y}_{t}\right) \triangleq \\
& \quad \triangleq A^{\mathbf{q}} X_{t}+\left(\gamma_{t}^{\mathbf{q}}\left(X_{t}\right)\right)^{\boldsymbol{T}} \mathbf{C}_{t}^{-1}\left(X_{t}\right)\left(\mathbf{y}_{t}-\boldsymbol{A}_{t} X_{t}\right),
\end{aligned}
$$

that is, the SSCP problem coincides with the problem of sequentially evaluating the optimal nonlinear filter of a particular functional of the state and the observations, $\phi_{t}(\cdot, \cdot)$. In this respect, the following result is true, providing a closed form
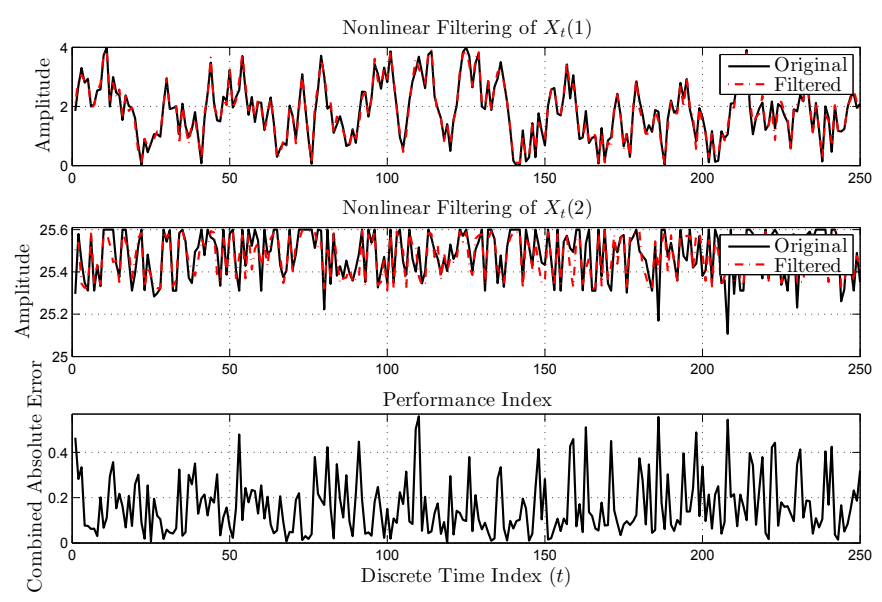

Figure 1. Demonstration of channel state tracking for 250 time steps.

approximate solution to the SSCP problem, at the same time enjoying asymptotic optimality in the sense of Theorem 1.

Theorem 2. (Approximate Solution to the SSCP Problem [13]) The strictly optimal spatiotemporal predictor of the channel magnitude at an arbitrary position $\mathbf{q} \in \mathbb{R}^{2}, \widehat{y}_{t}(\mathbf{q})$, can be approximated as

$$
\mathcal{E}^{L_{S}}\left(y_{t+\rho}(\mathbf{q}) \mid \mathscr{Y}_{t}\right) \triangleq\left\{\begin{array}{ll}
\left\langle\phi_{t}\left(\mathbf{y}_{t}\right), \frac{E_{t}}{\left\|E_{t}\right\|_{1}}\right\rangle, & \rho \equiv 0 \\
A^{\mathbf{q}} \mathbf{X} \boldsymbol{P}^{\rho} \frac{E_{t}}{\left\|E_{t}\right\|_{1}}, & \rho \geq 1
\end{array},\right.
$$

for all $t \in \mathbb{N}$, where the process $E_{t} \in \mathbb{R}^{L_{S} \times 1}$ can be recursively evaluated as in Theorem 1 and where the stochastic process $\phi_{t}\left(\mathbf{y}_{t}\right) \in \mathbb{R}^{L_{S} \times 1}$ is defined as

$$
\phi_{t}\left(\mathbf{y}_{t}\right) \triangleq\left[\phi_{t}\left(\boldsymbol{x}_{L_{S}}^{1}, \mathbf{y}_{t}\right) \ldots \phi_{t}\left(\boldsymbol{x}_{L_{S}}^{L_{S}}, \mathbf{y}_{t}\right)\right]^{\boldsymbol{T}},
$$

with $\phi_{t}: \mathbb{R}^{M \times 1} \times \mathbb{R}^{N \times 1} \mapsto \mathbb{R}$ defined as in (11). Additionally, under the same conditions as in Theorem 1, it is true that

$$
\sup _{\substack{t \in \mathbb{N}_{T} \\ \omega \in \widehat{\Omega}_{T}}}\left|\mathcal{E}^{L_{S}}\left(y_{t+\rho}(\mathbf{q}) \mid \mathscr{Y}_{t}\right)-\widehat{y}_{t+\rho}(\mathbf{q})\right|(\omega) \underset{L_{S} \rightarrow \infty}{\longrightarrow} 0 .
$$

\section{Numerical Simulations}

The practical effectiveness of the proposed estimators will also be validated through a number of sufficiently representative synthetic experiments. Specifically, we consider $N \equiv 30$ sensors randomly scattered on a square grid consisting of 3600 points, in the square region of the $x / y$ plane $\mathcal{S} \equiv[0,40]^{2}$ (in $m \times m)$. The position of the reference antenna is fixed at $\mathbf{p}_{\text {ref }} \equiv[2510]^{T}$. The variance of the multipath fading noise term is set at $\sigma_{\xi}^{2} \equiv 2$ and, for simplicity, we assume that the correlation distance is known and constant with respect to time and equal to $10 \mathrm{~m}$. As a result, in this simple example, the 
Spatial Channel Prediction at time $t$
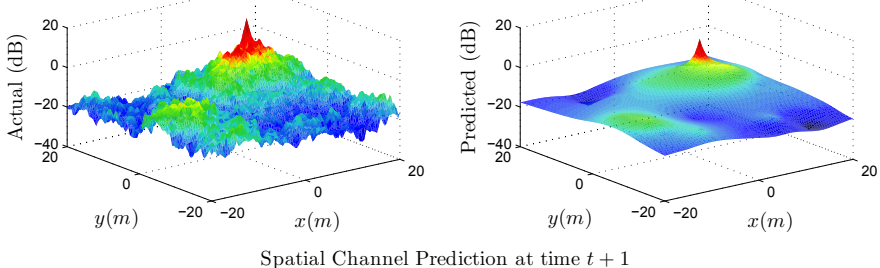

Spatial Channel Prediction at time $t+1$
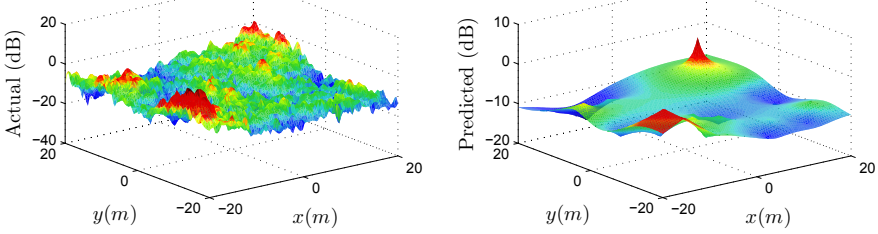

Figure 2. Spatial prediction and temporal tracking of the channel combined.

channel state is two dimensional, with $X_{t}(1) \equiv \mu\left(X_{t}\right)$ and $X_{t}(2) \equiv \eta^{2}\left(X_{t}\right)$ representing the path loss coefficient and the shadowing power, respectively. The evolution of the channel state is described by the stochastic difference equations

$$
\begin{aligned}
& X_{t}(1) \equiv \tanh \left(\gamma\left(X_{t-1}(1)-2\right)\right)+W_{t}+2, \quad \text { and } \\
& X_{t}(2) \equiv 0.3 \mid \tanh \left(\sin \left(\gamma X_{t-1}(2) W_{t}\right)+\right. \\
& \left.\quad+X_{t-1}(2) W_{t}\right)+W_{t} \mid+25, \quad \forall t \in \mathbb{N},
\end{aligned}
$$

for some arbitrary but known initial conditions, where $\gamma \equiv 1.6$ and $W_{t} \equiv \operatorname{clip}_{[-1,1]}\left(G_{t}\right), G_{t} \stackrel{i . i . d .}{\sim} \mathcal{N}(0,1)$, with $\operatorname{clip}_{[-1,1]}(\cdot)$ denoting the hard limiter operation into the set $[-1,1]$. Note that both difference equations are strongly nonlinear in both the state and driving noise. The state $X_{t} \equiv\left[X_{t}(1) X_{t}(2)\right]^{\boldsymbol{T}}$ was uniformly quantized into $L_{S} \equiv 30^{2}$ cells (that is, $L \equiv 30$ ). For simplicity, we focus on the case where $\rho \equiv 0$, that is, we consider the problems of temporal filtering of the channel state and spatial prediction of the channel, which both constitute instances of the SCST and SSCP problems, respectively.

Fig. 1 demonstrates the performance of channel state tracking (temporal filtering of the state) for 250 time steps. As illustrated in the figure, the quality of the estimates is excellent, considering the nonlinearity present both in the state process and the observations at each sensor in the network. It is also apparent that the produced estimation process behaves in a stable manner, as time increases. The filter of the channel state can subsequently be used for the also asymptotically optimal prediction of the channel magnitude in the rest of the space. This is illustrated in Fig. 2, where the combined spatial prediction and temporal tracking of the channel magnitudes are shown (for two time instants), in comparison to the real channel maps in the square region under consideration. From the figure, it can be seen that the quality of the predicted process is very good, especially considering the fact that the channel is reconstructed using only $0.83 \%$ of the total number of grid points in the region of interest. Of course, the quality of the spatial prediction improves as the number of spatial measurements (and therefore nodes/sensors) increases. One can observe that the prediction procedure accurately captures the basic characteristics of the channel in the region of interest, effectively exploiting the spatial correlations due to shadowing.

\section{Conclusion}

In this paper, a nonlinear filtering framework was proposed for addressing the fundamental problems of nonlinear sequential channel gain map tracking in mobile cooperative networks. Formulating the channel observations at the sensors as a HMM, a grid based approximate filtering scheme was employed for accurately tracking the temporal variation of the channel state, based on which a recursive spatiotemporal channel gain predictor was proposed, providing sequential $\mathrm{CG}$ map estimation at each sensor in the network. Further, we showed that both estimators are asymptotically MMSE optimal in a technically strong sense. In addition to these theoretical results, numerical simulations were presented, validating the practical effectiveness of the proposed approach.

\section{REFERENCES}

[1] N. Chatzipanagiotis, Y. Liu, A. Petropulu, and M. Zavlanos, "Distributed cooperative beamforming in multi-source multi-destination clustered systems," Signal Processing, IEEE Transactions on, vol. 62, no. 23, pp. 6105-6117, Dec 2014.

[2] L. Dong, Z. Han, A. Petropulu, and H. Poor, "Improving wireless physical layer security via cooperating relays," Signal Processing, IEEE Transactions on, vol. 58, no. 3, pp. 1875-1888, March 2010.

[3] A. Kaya, L. Greenstein, and W. Trappe, "Characterizing indoor wireless channels via ray tracing combined with stochastic modeling," Wireless Communications, IEEE Transactions on, vol. 8, no. 8, pp. 4165-4175, August 2009.

[4] N. Chatzipanagiotis, Y. Liu, A. Petropulu, and M. M. Zavlanos, "Controlling groups of mobile beamformers," in IEEE Conference on Decision and Control, Hawaii, 2012.

[5] D. S. Kalogerias, N. Chatzipanagiotis, M. M. Zavlanos, and A. P. Petropulu, "Mobile jammers for secrecy rate maximization in cooperative networks," in Acoustics, Speech and Signal Processing (ICASSP), 2013 IEEE International Conference on, May 2013, pp. 2901-2905.

[6] D. S. Kalogerias and A. P. Petropulu, "Mobi-cliques for improving ergodic secrecy in fading wiretap channels under power constraints," in Acoustics, Speech and Signal Processing (ICASSP), 2014 IEEE International Conference on, May 2014, pp. 1578-1591.

[7] S.-J. Kim, E. Dall'Anese, and G. Giannakis, "Cooperative spectrum sensing for cognitive radios using kriged kalman filtering," Selected Topics in Signal Processing, IEEE Journal of, vol. 5, no. 1, pp. 24 36, Feb 2011.

[8] E. Dall'Anese, S.-J. Kim, and G. Giannakis, "Channel gain map tracking via distributed kriging," Vehicular Technology, IEEE Transactions on, vol. 60, no. 3, pp. 1205-1211, March 2011.

[9] M. Malmirchegini and Y. Mostofi, "On the spatial predictability of communication channels," Wireless Communications, IEEE Transactions on, vol. 11, no. 3, pp. 964-978, March 2012.

[10] R. J. Elliott, L. Aggoun, and J. B. Moore, Hidden Markov Models. Springer, 1994.

[11] G. Pagès, H. Pham et al., "Optimal quantization methods for nonlinear filtering with discrete-time observations," Bernoulli, vol. 11, no. 5, pp. 893-932, 2005.

[12] A. Goldsmith, Wireless communications. Cambridge university press, 2005.

[13] D. S. Kalogerias and A. P. Petropulu, "Sequential channel state tracking \& spatiotemporal channel prediction in mobile wireless sensor networks," IEEE Transactions on Signal and Information Processing over Networks, Submitted in 2015.

[14] M. Gudmundson, "Correlation model for shadow fading in mobile radio systems," Electronics Letters, vol. 27, no. 23, pp. 2145-2146, Nov 1991.

[15] D. S. Kalogerias and A. P. Petropulu, "Grid-based filtering of Markoy processes revisited: Asymptotic optimality \& recursive estimation," to be submitted in 2015. Draft available at: http://eceweb1.rutgers.edu/ $\sim$ dkalogerias/.

[16] — "Asymptotically optimal discrete time nonlinear filters from stochastically convergent state process approximations," IEEE Transactions on Signal Processing, to be published in 2015. Extended version available at: http://arxiv.org/abs/1411.6719. 